\title{
Decreased expression of a phagocytic receptor Siglec-1 on alveolar macrophages in chronic obstructive pulmonary disease
}

\author{
Atsushi Tanno ${ }^{\dagger}$, Naoya Fujino ${ }^{1 * \dagger} \mathbb{D}$, Mitsuhiro Yamada ${ }^{1}$, Hisatoshi Sugiura', Taizou Hirano ${ }^{1}$, Rie Tanaka',
} Hirohito Sano', Satoshi Suzuki ${ }^{2}$, Yoshinori Okada ${ }^{3}$ and Masakazu Ichinose ${ }^{1}$

\begin{abstract}
Background: Alveolar macrophages are professional phagocytes that remove microbial pathogens inhaled into the lung. The phagocytic ability is compromised in chronic obstructive pulmonary disease (COPD). However, the molecular mechanisms underlying this defect in phagocytosis are not clearly defined.

Materials and methods: Cell suspensions were collected from lung tissues of patients undergoing lung resection. Alveolar macrophages were detected as $\mathrm{FSC}^{\text {hi }} / \mathrm{SSC}^{\text {hi }} / \mathrm{CD} 45^{+} / \mathrm{CD} 206^{+}$cells in the isolated cell suspension by flowcytometry. The cell surface expression of plasma membrane-bound phagocytic receptors (Fcy receptor I (FcyRl), a complement receptor CD11b, macrophage scavenger receptor-1 (MSR-1), CD36 and Siglec-1) was determined on the alveolar macrophages. Correlations between the expression levels of the phagocytic receptors and disease severity were analysed. Phagocytosis of fluorescence-tagged bacteria by human alveolar macrophages was evaluated.

Results: The flow-cytometry analyses revealed that FcyRl, CD11b, MSR-1 and Siglec-1, but not CD36, were expressed on human alveolar macrophages. Among these receptors, Siglec-1 expression was significantly decreased on alveolar macrophages in COPD ex-smokers $(n=11)$, compared to control never-smokers $(n=11)$ or control exsmokers $(n=9)$. The Siglec-1 expression on alveolar macrophages was significantly correlated with lung function (forced expiratory volume in $1 \mathrm{~s}$ ) and with the severity of emphysema. Treatment of human alveolar macrophages with an anti-Siglec1 blocking antibody decreased phagocytosis of non-typeable Haemophilus influenzae (NTHi).

Conclusion: Our findings demonstrated reduced expression of Siglec-1 on alveolar macrophages in COPD, which is involved in engulfment of NTHi.
\end{abstract}

Keywords: Chronic obstructive pulmonary disease, Alveolar macrophage, Phagocytosis, Siglec-1

\section{Background}

Alveolar macrophages serve as professional phagocytes that have the ability to eliminate inhaled microorganisms or noxious particles and endogenous apoptotic cells or cellular debris for innate host defence and the resolution of inflammation [1]. However, in the lung of patients with chronic obstructive pulmonary disease (COPD), alveolar macrophages participate in pathological processes

\footnotetext{
* Correspondence: nfujino@med.tohoku.ac.jp

${ }^{+}$Atsushi Tanno and Naoya Fujino contributed equally to this work.

${ }^{1}$ Department of Respiratory Medicine, Tohoku University Graduate School of

Medicine, 1-1 Seiryocho, Aobaku, Sendai 980 8574, Japan

Full list of author information is available at the end of the article
}

of chronic inflammation and tissue remodelling (i.e. alveolar destruction and small airway fibrosis) by producing reactive oxygen species (ROS), pro-inflammatory cytokines/chemokines, elastolytic proteases and profibrotic mediators [2, 3]. In addition to the dysregulated production of the mediators, recent studies have indicated that alveolar macrophages of COPD were functionally defective in the phagocytosis of microbial pathogens, such as Haemophilus influenzae [4-6], Streptococcus pneumoniae [5] and Moraxella catarrhalis [6]. This defective phagocytosis of microbes is implicated in the bacterial colonisation and persistent inflammation

(c) The Author(s). 2020 Open Access This article is distributed under the terms of the Creative Commons Attribution 4.0 International License (http://creativecommons.org/licenses/by/4.0/), which permits unrestricted use, distribution, and 
in the airway of COPD, which are postulated to cause acute exacerbations [7].

Phagocytosis is defined as the cellular uptake of particles $(>0.5 \mu \mathrm{m})$ by a plasma-membrane envelope and consists of multiple biological processes: recognition, engulfment, plasma-membrane fusion, maturation of phagosomes with progressive acidification and culminating in phagolysosomal fusion and digestion [8]. Plasma membrane-receptors have a critical role in the sensing and engulfment of microbes in the initial phase of phagocytosis and are classified into (i) opsonic receptors (Fc receptors and complement receptors) and (ii) nonopsonic, pattern-recognition receptors (scavenger receptors and lectin-like recognition molecules) [9]. Scavenger receptors such as macrophage scavenger receptor-1 (MSR-1, also known as SR-A1), macrophage receptor with collagenous structure (MARCO, also known as SRA2) and CD36 vary in the structure of their extracellular domains and recognise a large variety of molecules including not only microbial ligands (lipopolysaccharide and lipoteichoic acid) but also endogenous self-ligands (lipoproteins, unmodified proteins, etc.) [10]. Lectin-like recognition molecules also consist of diverse membranebound receptors such as C-type lectin receptors (mannose receptor or dectin-1, etc.) and sialic acid-binding immunoglobulin-like lectins (e.g. Siglec-1) [11]. This extremely diverse array of plasma membrane-receptors allows professional phagocytes to sense and eat a wide variety of foreign pathogens.

It is known that the expression of cell surface molecules is altered in alveolar macrophages of smokers or patients with COPD. Smoking reduces the expression of cell surface molecules involved in apoptotic cellclearance (CD91) and cellular adhesion (CD31, CD44) [12]. Moreover molecules involved in antigen presentation (CD86 and CD11a) are down-regulated in the alveolar macrophages of COPD [13]. Despite the increasing evidence of the phenotypic alteration of alveolar macrophages under a pathological condition, the expression levels of receptors mediating bacterial phagocytosis have not been clearly determined.

In this study, we aimed to determine alteration of expression levels of phagocytosis-associated receptors in COPD and its functional consequences in bacterial uptake. Here we analysed representative membrane-bound receptors that are known to be involved in bacteria phagocytosis including Fc $\gamma$ receptor I (Fc $\gamma \mathrm{RI}), \mathrm{CD} 11 \mathrm{~b}$ (a subset of a complement receptor), MSR-1, CD36 and Siglec-1 (also knonwn as CD169). We directly analysed the cell surface expression of these receptors on alveolar macrophages freshly isolated from human lung tissues using flow-cytometry and found that Siglec-1 expression was significantly decreased in alveolar macrophages isolated from lung tissues of patients with COPD.
Furthermore we confirmed that a blocking antibody for Siglec-1 reduced the phagocytosis capability of nontypeable Haemophilus influenzae in human alveolar macrophages.

\section{Methods}

\section{Patient population}

Subjects for the flow-cytometric analysis and immunostaining of phagocytic receptor expression participated in the study from September 2016 to August 2017. Subjects for the phagocytosis study participated between January 2018 to August 2019. All patients with COPD satisfied the Global Initiative for Chronic Obstructive Lung Disease (GOLD) guideline criteria [14]. Control ex-smokers and COPD ex-smokers had ceased smoking for more than 2 months before the enrolment. We excluded current smokers and subjects with asthma, bronchiectasis, pulmonary fibrosis or other inflammatory lung diseases. Written informed consent was obtained from all subjects who participated in this study. This study was approved by the ethics committees at Tohoku University School Graduate of Medicine (registration number: 2017-1-352) and Japanese Red Cross Ishinomaki Hospital (registration number: 13-12).

\section{Chest CT assessment of low attenuation areas}

We evaluated low attenuation area scores using a highresolution CT scan as an indicator for pulmonary emphysema according to the Goddard classification [15]. Briefly low attenuation value was scored for the bilateral upper, middle and lower lung fields. Zero represented no emphysema; 1 was given for up to $25 \%, 2$ for up to $50 \%, 3$ for up to $75 \%$ and 4 for total involvement or almost total absence of normal lung tissues. Scores ranged from 0 to 24 .

\section{Preparation of lung cell suspension}

Lung tissues were provided by patients who underwent lung resection due to primary lung cancer. We verified that the tissues had no pathological findings of tumours or overt fibrosis. Resected lung tissues were immediately immersed in StemSurvive-Lung tissue preservation solution (Kurabo Industries Ltd., Osaka, Japan), stored at $4{ }^{\circ} \mathrm{C}$ and used within 12 hours after surgery.

\section{Flow-cytometry analysis, fluorescence-activated cell sorting and diff-Quik staining}

We collected lung hematopoietic cells including alveolar macrophages by perfusing and lavaging tissues with saline. Detailed methods for flow-cytometric analyses were described in the online supplement. 


\section{Preparation of heat-killed and fluorescent dye-tagged non-typeable Haemophilus influenzae}

Non-typeable Haemophilus influenzae (NTHi; GTC 15013, JNBP_02694) was obtained from Gifu University Center for Conservation of Microbial Genetic Resource. The number of bacteria was counted with Bacteria Counting Kit (Thermo Fisher Scientific). NTHi was killed at $65^{\circ} \mathrm{C}$ for 1 hour and was labeled with pHrode Phagocytosis Particle Labeling Kit for Flow Cytometry (Thermo Fisher Scientific) according to the manufacturer's instruction.

\section{Phagocytosis assay}

Lung tissue was perfused with normal saline. The retrieved cell suspension was centrifuged at $400 \mathrm{~g}$ for 30 $\mathrm{min}$ at $20^{\circ} \mathrm{C}$. Cellular fractions in the cell suspensions were routinely evaluated by Diff-Quik staining. The cell pellet was resuspended in PBS and subjected to FicollPaque (GE Healthcare) density gradient centrifugation to remove granulocytes and erythrocytes. The mononuclear cell layer at the interface, mainly containing alveolar macrophages, was collected and washed with PBS. $6 \times$ $10^{5}$ cells were reconstituted into $2 \mathrm{~mL}$ of HEPESbuffered RPMI 1640 (Thermo Fisher Scientific) containing $1 \%$ IgG/protease-free BSA. $3 \times 10^{4}$ cells in $100 \mu \mathrm{L}$ of the cell suspension were transferred into each well of a 96 well formatted plate and incubated for 2 hours in the $5 \% \mathrm{CO}_{2}$ incubator at $37^{\circ} \mathrm{C}$. Anti-human Siglec-1 blocking antibody (BIO-RAD, clone 7-239, cat\# MCA2517EL) or an isotype-matched control antibody (mouse $\mathrm{IgG}_{1}$; BIO-RAD, cat\# MCA 928EL) was added and incubated for 1 hour at room temperature in order to block Siglec-1 on the surface of alveolar macrophages [16]. The final concentration of these antibodies was $10 \mu \mathrm{g} / \mathrm{mL}$, which was sufficient to block binding of additional phycoerythrin-conjugated anti-human Siglec-1 antibody (eBioscience, clone 7-239, cat\# 12-1699-42). This concentration of the antibody was not influential in cell viability (data not shown). Heat-killed and pHrodelabeled NTHi at $100 \mathrm{MOI}$ was added onto the culture media. After 1 hour, cells were collected with 0.5\% Trypsin/EDTA. Positive cells that engulfed pHrode-labeled bacteria in acidic phagosome compartments were quantified by flow-cytometry using FACS CantoII (BD Biosciences). Data were analysed by FlowJo (Tree Star Inc., OR, USA).

\section{Statistics}

All data are expressed as medians (with interquartile ranges) unless otherwise indicated. Statistical tests were performed using GraphPad Prism 8 (GraphPad Software Inc., CA, USA). Difference in the distribution of the genders between CNS, CES and COPD were analysed using the Chi square test. For comparison between three groups, the Kruskal-Wallis test followed by the Dunn's multiple comparison test was performed. Statistical correlation analyses were performed using Spearman's test. Multivariable regression analyses were performed for evaluating the effect of potentially confounding factors. $P$-values less than 0.05 were considered significant.

\section{Results}

Characterisation of phagocytosis-associated receptor expression on alveolar macrophages isolated from lung tissues of patients with COPD

To examine cell surface expression levels of phagocytosis-associated receptors on human alveolar macrophages, we collected cell suspension by perfusing and lavaging peripheral lung tissues with saline and delineated alveolar macrophages as $\mathrm{FSC}^{\text {hi }} / \mathrm{SSC}^{\text {hi }} / \mathrm{CD} 45^{+} /$ $\mathrm{CD}^{206^{+}}$cells in flow-cytometry according to previous studies [2, 3] (Fig. 1a). We confirmed that FSC ${ }^{\text {hi }} /$ SSC $^{\text {hi }}$ / $\mathrm{CD} 45^{+} / \mathrm{CD} 206^{+}$cells from human lung tissue lavage were alveolar macrophages in three ways. First, cytospin samples of sorted $\mathrm{FSC}^{\mathrm{hi}} / \mathrm{SSC}^{\mathrm{hi}} / \mathrm{CD} 45^{+} / \mathrm{CD} 206^{+}$cells exhibited homogenous, basophilic large cells that were morphologically consistent with alveolar macrophages (Additional file 1: Figure S1A). Second, immunohistochemistry verified that CD206 was expressed by alveolar macrophages in human lung sections and its expression pattern was not different between control never-smokers (CNS), control ex-smokers (CES) and COPD ex-smokers (COPD) (Additional file 1: Figure S1B). Third, $\mathrm{FSC}^{\text {hi }}$ / $\mathrm{SSC}^{\mathrm{hi}} / \mathrm{CD} 45^{+} / \mathrm{CD} 206^{+}$cells expressed CD14 at a minimum level, which was consisting with the previous reports [2, 3] (Additional file 1: Figure S1C).

We then examined the expression of each of phagocytosis-associated receptors (FcyRI, CD11b, MSR-1, CD36 and Siglec-1) on the surface of FSC ${ }^{\text {hi }}$ / $\mathrm{SSC}^{\mathrm{hi}} / \mathrm{CD} 45^{+} / \mathrm{CD}^{206^{+}}$alveolar macrophages in flowcytometry, that were collected from lung tissues of CNS $(n=11)$, CES $(n=9)$ and COPD with mild-tomoderate airflow limitation severity $(n=5$ in GOLD stage1, $n=6$ in GOLD stage2). The patient characteristics in the flow-cytometric analysis are shown in Table 1. We evaluated the receptor expression by two parameters: (i) the percentage of alveolar macrophages expressing each of the receptors to total alveolar macrophages and (ii) the geometric mean of the receptor expression intensity normalised by that of a corresponding fluorochrome minus one (FMO) control with an appropriate isotype control antibody. We found that FcyRI, CD11b, MSR-1 and Siglec-1 were expressed by alveolar macrophages isolated from CNS but CD36 exhibited no or little expression (Fig. 1b). The percentage of alveolar macrophages expressing Fc $\gamma$ RI or Siglec-1 was significantly decreased in COPD 


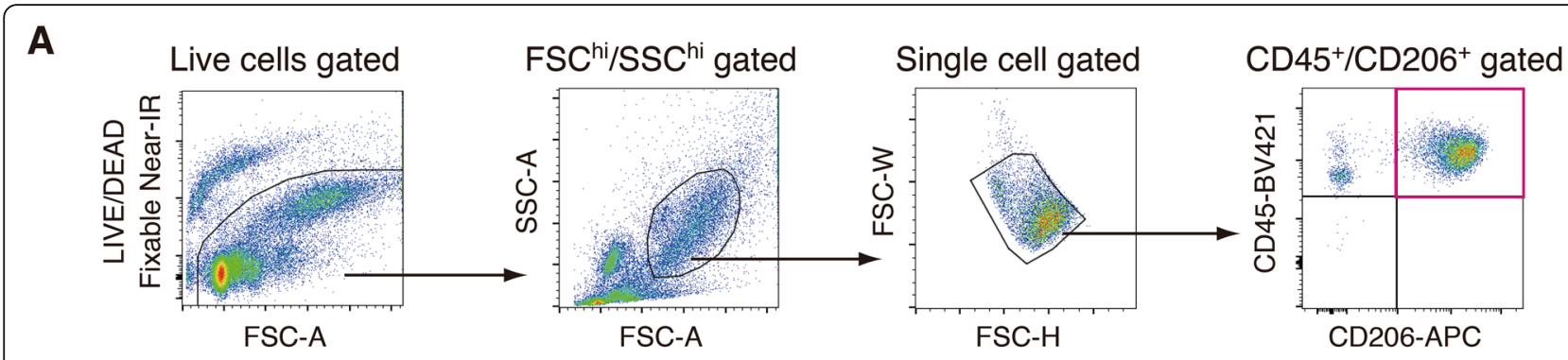

B

\section{$\mathrm{FSC}^{\text {hi }} / \mathrm{SSC}^{\mathrm{hi}} / \mathrm{CD} 45^{+} / \mathrm{CD} 206^{+}$- gated}
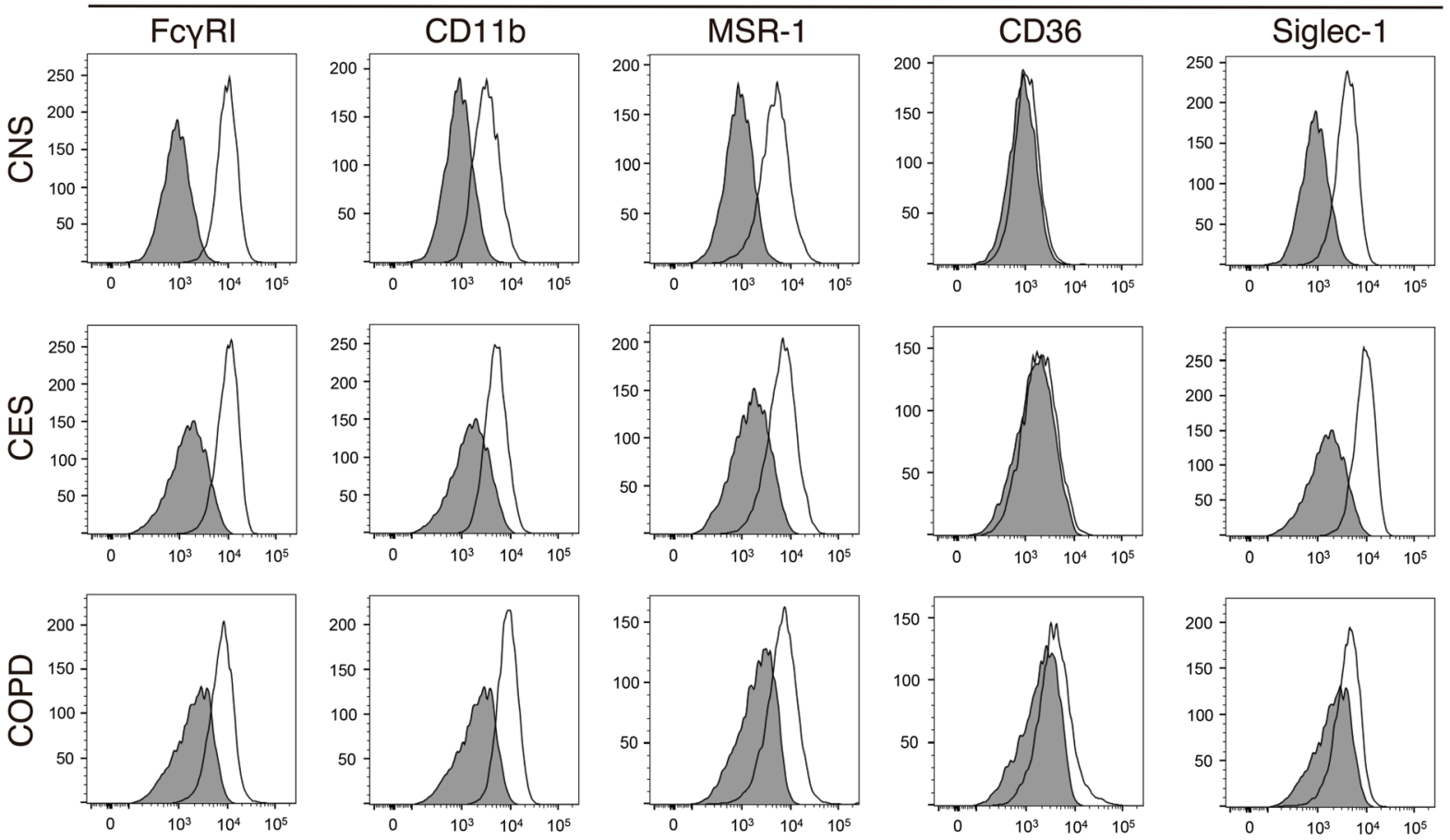

Fig. 1 Expression levels of phagocytic receptors on human alveolar macrophages. a FACS gating strategy to delineate alveolar macrophages. Cell suspension was collected by perfusing and lavaging human lung tissues with saline. Alveolar macrophages were detected as live, FSChi/SSChi, single cell-gated $\mathrm{CD}_{4} 5^{+} / \mathrm{CD} 206^{+}$cells in the cell suspension. b Representative histograms for the expression levels of FcyRl, CD11b, macrophage scavenger receptor-1 (MSR-1), CD36 and Siglec-1, on FSC Chi/SSChi/CD45 $/$ CD206 ${ }^{+}$alveolar macrophages of control never-smokers $(C N S, n=11)$, control ex-smokers (CES, $n=9$ ) and COPD ex-smokers (COPD, $n=11$ ). Specific staining, open; isotype control, shaded

subjects compared to CNS and CES (Fig. 2a). Moreover the percentage of alveolar macrophages expressing MSR-1 was slightly decreased in COPD subjects compared to CES, although its decrease was significant between COPD and CNS (Fig. 2a). Among these receptors, the reduction of Siglec-1 expression intensity on alveolar macrophages of COPD patients was statistically significant compared to CNS and CES (Fig. 2b). Although the difference in the expression intensity of FCYRI and MSR-1 of between CES and COPD was not statistically significant, the intensity of these receptors was decreased in COPD compared to CNS (Fig. 2b).
Relationship between phagocytosis-associated receptor expression on alveolar macrophages and clinical variables Considering the differences in the expression level of Fc $\gamma$ RI, MSR-1 and Siglec-1 on alveolar macrophages between CNS, CES and COPD, we next sought to determine whether these differences were associated with clinical variables such as age, gender, smoking history, airflow limitation severity and pulmonary emphysema. The severity of airflow limitation was evaluated as \% predicted values of forced expiratory volume in 1 second ( $\mathrm{FEV}_{1} \%$ pred). Pulmonary emphysema was quantified by measuring low attenuation areas on chest computed 
Table 1 Patient characteristics in flow-cytometry analyses and immunohistochemistry results of phagocytic receptor expression of alveolar macrophages

\begin{tabular}{|c|c|c|c|}
\hline & Control never-smokers & Control ex-smokers & COPD ex-smokers \\
\hline Subjects (n) & 11 & 9 & 11 \\
\hline Men/women & $1 / 10$ & $7 / 2^{* * *}$ & $11 / 0^{* * * *}$ \\
\hline Age & $72(64.0-77.0)$ & $75(65.5-77.5)$ & $67(64.0-73.0)$ \\
\hline Smoking (pack-years) & $0.0(0.0-0.0)$ & $33.0(18.3-49.1)^{* *}$ & $43.0(30.0-68.8)^{* * * *}$ \\
\hline Duration of smoking cessation (months) & NA & $48.0(2.0-168.0)$ & $12.0(2.0-120.0)$ \\
\hline FVC (\%pred) & $100.7(94.1-123.5)$ & $95.2(86.7-106.9)$ & $112.0(101.5-123.3)$ \\
\hline $\mathrm{FEV}_{1}$ (\%pred) & $104.7(98.6-119.4)$ & $90.5(83.0-94.7)$ & $78.7(66.8-93.4)^{* * *}$ \\
\hline $\mathrm{FEV}_{1} / \mathrm{FVC}(\%)$ & $80.7(74.9-85.2)$ & $76.3(73.5-78.7)$ & $62.6(54.0-64.7)^{* * * *}+\dagger$ \\
\hline LAA score & $0(0-0)$ & $0(0-0)$ & $5(4-9) * * *+\dagger \dagger$ \\
\hline GOLD Grade (1/2/3/4) & NA & NA & $5 / 6 / 0 / 0$ \\
\hline Exacerbations per year & NA & NA & 0 \\
\hline \multicolumn{4}{|l|}{ Treatment } \\
\hline$\angle A B A$ & NA & NA & 1 \\
\hline LAMA & NA & NA & 5 \\
\hline LABA/LAMA & NA & NA & 0 \\
\hline ICS & NA & NA & 0 \\
\hline ICS/LABA & NA & NA & 0 \\
\hline ICS/LABA/LAMA & NA & NA & 0 \\
\hline Theophylline & NA & NA & 0 \\
\hline Viable cell number per gram tissue ${ }^{a}$ & $7.4 \times 10^{6}\left(4.9 \times 10^{6}-1.3 \times 10^{7}\right)$ & $1.1 \times 10^{7}\left(7.5 \times 10^{6}-2.3 \times 10^{7}\right)$ & $1.1 \times 10^{7}\left(8.8 \times 10^{6}-1.9 \times 10^{7}\right)$ \\
\hline Viability $(\%)^{b}$ & $88(83-91)$ & $90(85-92)$ & $88(85-92)$ \\
\hline
\end{tabular}

Data are presented as the median (with interquartile ranges). Data were analysed with Chi-square test, Kruskal-Wallis test followed by Dunn's multiple comparison test or Mann-Whitney test

aiable cell number per gram tissue, the number of whole viable cells that were collected from 1 gram of lung tissues

${ }^{b}$ Viability was evaluated with trypan blue staining

${ }^{* *} P<0.01,{ }^{* * *} P<0.001,{ }^{* * * *} P<0.0001$ compared with control never-smokers; $\dagger+P<0.01,+\dagger+P<0.001$ compared with control ex-smokers

FVC forced vital capacity; $F E V_{1}$, forced expiratory volume in $1 \mathrm{~s} ; F E V$ \%pred \% predicted values of FEV ; $_{1}$ LAA, low attenuation area; GOLD Global Initiative for Chronic Obstructive Lung Disease; LABA long-acting $\beta$ agonists; LAMA long-acting muscarinic antagonists; ICS inhaled corticosteroids; NA not applicable

tomography $(\mathrm{CT})$ images. We found that smoking history, $\mathrm{FEV}_{1} \%$ pred and low attenuation area scores exhibited a significant association with the phagocytosisassociated receptor expression (Table 2). A multiple linear regression analysis revealed that the severity of pulmonary emphysema was independently associated with the reduction of Siglec-1 expression on alveolar macrophages (Table 3).

\section{Siglec-1 was important for engulfment of non-typeable} Haemophilus influenzae by human alveolar macrophages So far we observed the significant reduction of Siglec1 protein expression on alveolar macrophages of patients with COPD. Siglec-1 is known to recognise sialylated bacteria such as Neisseria meningitidis [17]. Considering that non-typeable Haemophilus influenzae (NTHi) was a sialylated pathogen and was mainly associated with exacerbation and bacterial colonisation of COPD, we therefore sought to determine whether Siglec-1 was functionally important for uptake of
NTHi by human alveolar macrophages. As we wished to evaluate how Siglec-1 affected engulfment at the initial stage of bacterial phagocytosis, but not microbicidal efficacy in the late stage, we used heat-killed, fluorescent dye-tagged NTHi that increased in fluorescence under an acidic condition in phagolysosomes. We evaluated alveolar macrophages isolated from control never smokers that expressed Siglec-1 as shown by immunohistochemistry (Additional file 1: Figure S2). The patients' characteristics was shown in Table 4 . Cellular fractions of collected cell suspensions were as follows: macrophages, $89.0 \pm 2.3 \%$; lymphocytes, $6.2 \pm$ $2.0 \%$; neutrophils, $3.8 \pm 1.5 \%$; eosinophils, $0.4 \pm 0.3 \%$; monocytes, $0.8 \pm 0.3 \%$. After density gradient centrifugation to remove granulocytes, mononuclear cells were seeded on culture plates, most of which were thought to be alveolar macrophages. Then, adherent cells were cultured in the presence of an anti-Siglec-1 blocking antibody to inhibit biding of bacteria to Siglec-1 or an isotype-matched control antibody, which was followed 
A
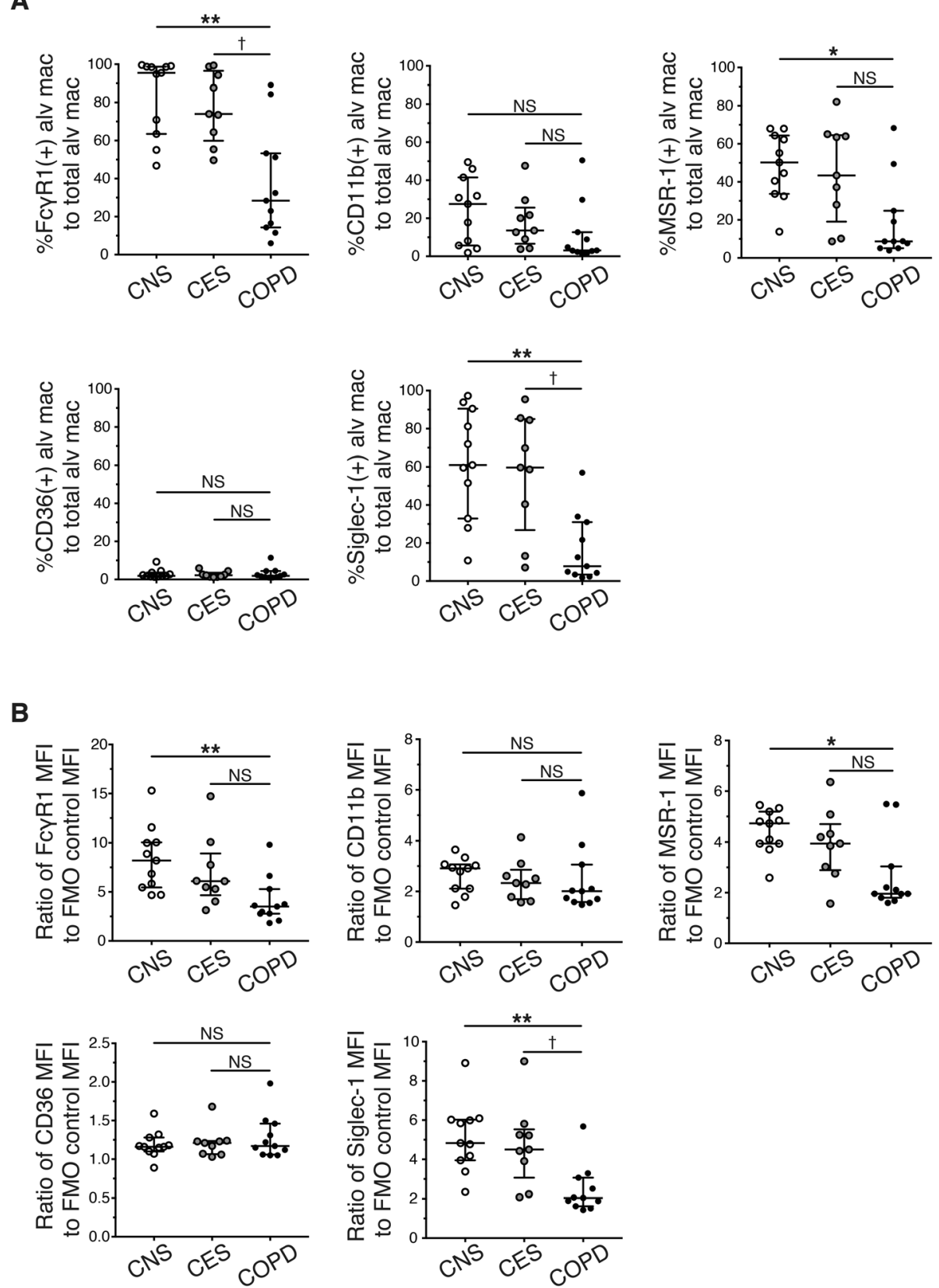

Fig. 2 A decrease in cell surface expression of Siglec-1 on alveolar macrophages isolated from COPD lungs. Each antigen expression is shown as (a) the percentage of positive alveolar macrophages expressing each phagocytic receptor to the total alveolar macrophages and (b) the ratio of the geometric mean of the phagocytic receptor expression levels to the geometric mean of the corresponding isotype control in control neversmokers (CNS, $n=11$, white circles), control ex-smokers (CES, $n=9$ grey circles) and COPD ex-smokers (COPD, $n=11$, black circles). Data represent median with interquartile ranges and were analysed with Kruskal-Wallis test followed by Dunn's Multiple Comparison Test. ${ }^{*} P<0.05$, ${ }^{* *} P<0.01$ compared with CNS; †P $<0.05$ compared with CES. N.S. indicates "not significant"

by incubation with fluorescent dye-labeled NTHi. A flow-cytometry analysis indicated that the anti-Siglec-1 blocking antibody significantly reduced the percentage of NTHi-engulfed macrophages or the mean fluorescence intensity in macrophages (Fig. 3).

\section{Discussion}

Our data suggested two novel aspects for human alveolar macrophages which may explain the compromised phagocytic ability of the alveolar macrophages in COPD; (i) the extracellular expression of plasma membrane- 
Table 2 Correlation of clinical variables and phagocytosis-associated receptor expression on alveolar macrophages (univariable analysis)

\begin{tabular}{|c|c|c|c|c|c|c|c|}
\hline & & \multicolumn{2}{|l|}{ FcyRl } & \multicolumn{2}{|l|}{ MSR-1 } & \multicolumn{2}{|l|}{ Siglec-1 } \\
\hline & & MFI ratio & $\%$ positive & $\mathrm{MFI}$ & $\%$ positive & $\mathrm{MFI}$ & $\%$ positive \\
\hline \multirow[t]{2}{*}{ Age } & $r_{s}$ & 0.2735 & 0.1550 & 0.1842 & 0.1776 & 0.3462 & 0.3061 \\
\hline & $P$ value & 0.1365 & 0.4051 & 0.3213 & 0.3392 & 0.0564 & 0.0940 \\
\hline \multirow[t]{2}{*}{ Gender } & $r_{s}$ & -0.2666 & -0.3258 & -0.1852 & -0.2889 & -0.3184 & -0.3184 \\
\hline & $P$ value & 0.0737 & 0.3185 & 0.1149 & 0.0809 & 0.0809 & 0.0737 \\
\hline \multirow[t]{2}{*}{ Smoking history (Pack-years) } & $r_{s}$ & -0.3670 & -0.3645 & -0.3568 & -0.3817 & -0.5333 & -0.4753 \\
\hline & $P$ value & 0.0423 & 0.0438 & 0.0488 & 0.0341 & 0.0020 & 0.0069 \\
\hline \multirow[t]{2}{*}{$\mathrm{FEV}_{1}$ (\% pred) } & $r_{s}$ & 0.4140 & 0.3533 & 0.5115 & 0.4459 & 0.4656 & 0.3958 \\
\hline & $P$ value & 0.0206 & 0.0512 & 0.0033 & 0.0119 & 0.0083 & 0.0275 \\
\hline \multirow[t]{2}{*}{ Low attenuation area scores } & $r_{s}$ & -0.5358 & -0.5577 & -0.4564 & -0.4945 & -0.6778 & -0.6440 \\
\hline & $P$ value & 0.0019 & 0.0011 & 0.0099 & 0.0047 & $<0.0001$ & $<0.0001$ \\
\hline
\end{tabular}

Bold denotes values $P<0.05$

$r_{s}$ Spearman rank correlation coefficient. $\mathrm{FEV}_{1}$, forced expiratory volume in 1 second. MFI, mean fluorescence intensity MFI ratio indicates the ratio of receptor MFI to the corresponding FMO control MFI

$\%$ positive indicates the percentage of a receptor-positive alveolar macrophages to total alveolar macrophages

bound receptor for phagocytosis, Siglec-1, could be altered under pathological conditions in COPD lung and (ii) Siglec-1 was at least partially involved in the engulfment of NTHi by human alveolar macrophages. Importantly we observed reduced Siglec-1 expression in mildto-moderate COPD subjects who had never experienced exacerbations, indicating that phagocytic receptor expression could be altered in early stages of COPD.

Siglecs (sialic acid-binding immunoglobulin (Ig)-like lectins) are type 1 membrane proteins containing a homologous N-terminal V-set Ig-like domain that recognises sialylated glycan, followed by variable numbers of $\mathrm{C} 2$ set domains [18]. The Siglec family is classified into two subfamilies: (i) Siglecs that are conserved across mammals (Siglec-1, CD22, myelin-associated glycoprotein and Siglec-15) and (ii) CD33-related Siglecs that are variable across mammals and therefore are postulated to have evolved rapidly [17]. Most Siglecs except for Siglec-
1 have cytoplasmic regions that mediate intracellular signalling and regulate innate and adaptive immunity. CD22 and most CD33-related Siglecs have immunoreceptor tyrosine-based inhibitory motifs (ITIMs) in the cytoplasmic domains [18], that suppress activation signals by recruiting cytoplasmic phosphatases containing a Src homology 2 (SH2) domain [19]. Conversely a few Siglecs, such as Siglec-14, coupled with immunoreceptor tyrosine-based activating motifs (ITAMs)-bearing adaptors have the ability to activate immune cells via spleen tyrosine kinase (Syk) [17].

Some emerging evidence from human genetic studies has suggested that Siglecs are involved in pathogenesis of COPD. Null allele of SIGLEC14 encoding immuneactivating Siglec-14 was associated with a reduced risk of COPD exacerbation, possibly via suppression of proinflammatory responses by inactivation of Syk [20]. An integrative genomics approach utilising single nucleotide

Table 3 Correlation of clinical variables and phagocytosis-associated receptor expression of alveolar macrophages (multiple linear regression analysis)

\begin{tabular}{|c|c|c|c|c|c|c|c|}
\hline & & \multicolumn{2}{|l|}{ FcyRl } & \multicolumn{2}{|l|}{ MSR-1 } & \multicolumn{2}{|l|}{ Siglec-1 } \\
\hline & & MFI ratio & $\%$ positive & MFI & $\%$ positive & MFI & $\%$ positive \\
\hline \multirow[t]{2}{*}{ Smoking history (Pack-years) } & Estimate & 0.0116 & -0.1288 & 0.0105 & 0.0514 & -0.0193 & -0.2747 \\
\hline & $P$ value & 0.7078 & 0.6213 & 0.2396 & 0.8057 & 0.2488 & 0.2935 \\
\hline \multirow[t]{2}{*}{$\mathrm{FEV}_{1}(\%$ pred $)$} & Estimate & 0.0192 & 0.1153 & 0.0297 & 0.3519 & -0.0057 & -0.1107 \\
\hline & $P$ value & 0.7158 & 0.7946 & 0.0548 & 0.3265 & 0.8397 & 0.8016 \\
\hline \multirow[t]{2}{*}{ Low attenuation area scores } & Estimate & -0.4466 & -3.8890 & -0.0002 & -2.6940 & -0.2661 & -5.0410 \\
\hline & $P$ value & 0.0731 & 0.0637 & 0.9982 & 0.1070 & 0.0463 & 0.0180 \\
\hline
\end{tabular}

Estimate represents parameter estimate

Bold denotes values $P<0.05$

$\mathrm{FEV}_{1}$, forced expiratory volume in one second. MFI, mean fluorescence intensity

$\mathrm{MFI}$ ratio indicates the ratio of receptor MFI to the corresponding FMO control MFI

$\%$ positive indicates the percentage of a receptor-positive alveolar macrophages to total alveolar macrophages 
Table 4 Patient characteristics in the phagocytosis assay

\begin{tabular}{ll}
\hline & Control never-smokers \\
\hline Subjects (n) & 5 \\
Men/women & $0 / 5$ \\
Age & $72.5(65.8-74.8)$ \\
Smoking (pack-years) & $0.0(0.0-0.0)$ \\
FVC (\%pred) & $114.7(107.4-122.6)$ \\
FEV $_{1}(\%$ pred) & $116.6(113.1-119.8)$ \\
FEV $_{1} /$ FVC (\%) & $80.9(75.7-83.0)$ \\
Viable cell number per gram tissue $^{\mathrm{a}}$ & $3.1 \times 10^{6}\left(2.9 \times 10^{6}-3.8 \times 106\right)$ \\
Viability (\%) $^{\text {b }}$ & $88(77-90)$ \\
\hline
\end{tabular}

Data are presented as the median (with interquartile ranges)

aviable cell number per gram tissue, the number of whole viable cells that were collected from one gram of lung tissues

${ }^{b}$ Viability was evaluated with trypan blue staining

FVC forced vital capacity; FEV 1 forced expiratory volume in $1 \mathrm{~s} ; F E V_{1} \%$ pred $\%$ predicted values of $\mathrm{FEV}_{1}$ polymorphisms (SNPs) on COPD phenotypes and a genome-wide expression quantitative trait loci (eQTLs) study on lung tissues revealed that CD22 was one of potential causal genes for airflow limitation in patients with COPD [21]. However, to our knowledge, there has been no report showing a relationship between Siglec-1 and COPD.

Siglec-1 (also known as sialoadhesin or CD169) preferentially binds to $\alpha 2,3-$ linked sialic acids $[22,23]$ and has longer extracellular regions (i.e., 17 Ig domains) that lack intracellular signalling motifs [24]. This unique structure of Siglec-1 may enable extension of sialic acid-binding sites outside the plasma membranes [25] and probably is useful for recognition, capture and uptake of microbes [17]. Siglec-1 is expressed by tissue macrophages and has a critical role in recognition and clearance of sialylated bacterial pathogens such as Neisseria meningitidis

\section{A}

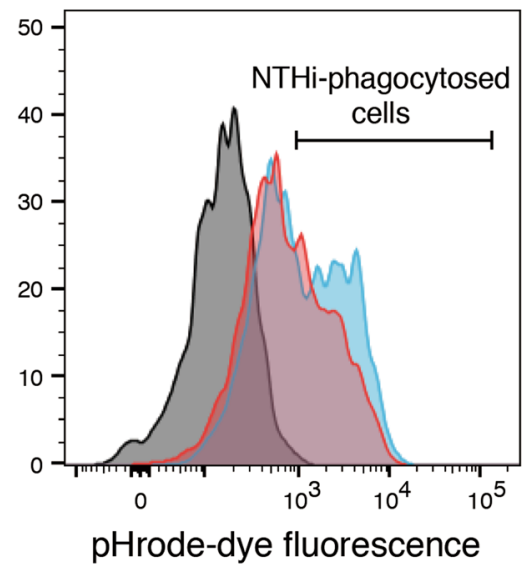

$\square$ No pHrode-labeled NTH

pHrode-labeled NTHi

+ isotype-matched control antibody

pHrode-labeled NTH

+ anti-human Siglec-1 blocking antibody
B
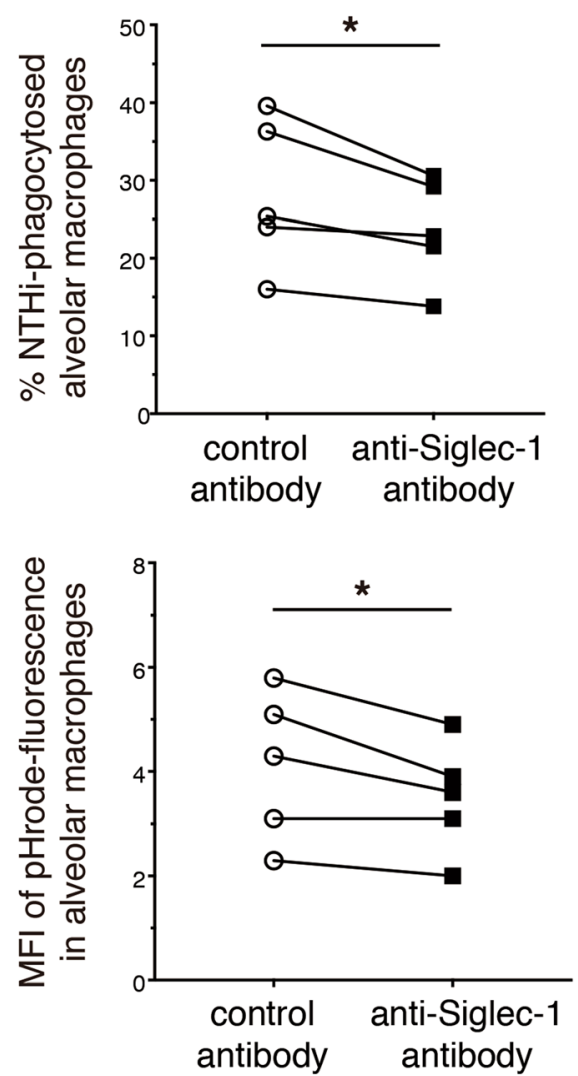

Fig. 3 An anti-human Siglec-1 blocking antibody reduced engulfment of NTHi by human alveolar macrophages ex vivo. a Alveolar macrophages isolated from resected lung tissues of control never smokers were incubated with heat-killed and pHrode dye-labeled NTHi in the presence of an anti-human Siglec-1 blocking antibody or an isotype-matched control antibody. The bacterial engulfment was evaluated by flowcytometry. A representative histogram of pHrode-dye fluorescence was shown. A grey line indicated cells without pHrode-labeled NTHi as a negative control. A blue line showed cells treated with pHrode-labeled NTHi in the presence of an isotype-matched control antibody. A red line represented cells treated with pHrode-labeled NTHi in the presence of an anti-human Siglec-1 blocking antibody. $\mathbf{b}$ Bacterial engulfment was quantified by the percentage of NTHi-phagocytosed alveolar macrophages (upper panel) or by the mean fluorescence intensity (MFI) of pHrode-fluorescence in alveolar macrophages (lower panel). Anti-Siglec-1 antibody treatment reduced the engulfment ability $\left(N=5,{ }^{*} P<0.05\right.$, Paired t-test) 
[26], Campyrobacter jejuni [27] and group B streptococcus [28]. In addition, Siglec-1 was required for proper pro-inflammatory responses via tumour necrosis factor$\alpha$ and interferon (IFN) $\beta$ to eliminate C.jejuni that was intravenously administered to mice [29]. NTHi is also known to be a sialylated microbe and has genes encoding $\alpha 2,3$-sialyltransferase which adds sialic acids onto its lipooligosaccharide [30,31]. Considering a number of evidence that NTHi is one of the most common bacteria involved in bacterial colonisation and acute exacerbation of COPD [32, 33], our data suggest that the reduced capability of alveolar macrophage phagocytosis for NTHi in COPD is due to the decrease in the extracellular expression of Siglec-1.

Although accumulating studies observed the defective phagocytosis of microbial pathogens including NTHi and S.pneumoniae by alveolar macrophages in COPD [4-6], there are few reports that identified molecular mechanisms involved in the reduced phagocytic ability. Bewley et al. recently reported that up-regulation of the anti-apoptotic protein Mcl-1 and a failure to produce mitochondrial ROS in alveolar macrophages of COPD reduced intracellular killing of S.pneumoniae [34]. In addition Harvey et al. reported that Nrf2 activation improved the phagocytic ability for NTHi by increasing the scavenger receptor MARCO in alveolar macrophages derived from patients with COPD, though it has not been examined whether MARCO protein expression is down-regulated on the surface of alveolar macrophages of COPD compared to control smokers [35]. Thus our study firstly showed the possibility that an alteration of plasma membrane-bound phagocytic receptors might be a critical indicator for bacterial clearance of alveolar macrophages under inflammatory conditions.

The multivariable linear regression analysis revealed that the severity of pulmonary emphysema evaluated by the low attenuation area score on chest CT scans was an independent parameter associated with the decrease in Siglec- 1 on alveolar macrophages. Although type 1 interferon was known to induce Siglec-1 protein expression in patients with systemic sclerosis [36] or with human immunodeficiency virus-1 [37], it has not been determined whether and how the Siglec-1 protein expression is down-regulated during pathological processes. Thus our finding provides new evidence of the reduced expression of Siglec-1 in COPD of which pathogenesis is involved in chronic inflammation related to excessive oxidative stress and would provide a rationale to investigate molecular basis of upstream signalling pathways negatively regulating Siglec-1 expression.

A large number of studies support the idea that Siglec1-expressing tissue macrophages have critical roles in initiation of proper pro-inflammatory responses to maintain homeostasis in viral infection, cancer and autoimmune diseases $[11,17,38]$. This idea was further validated by specific depletion of Siglec-1-positive macrophages using Siglec-1-diphteria toxin receptortransgenic mice [39, 40]. Taken together with these reports, our observation suggests that Siglec-1 $1^{\text {dim-neg }}$ alveolar macrophages in COPD lungs might represent a dysfunctional macrophage for innate host defence.

There are some limitations in our study. First, the sample size of our flow-cytometric analysis was small with only mild and moderate COPD, because the availability of resected lung tissues, especially from patients with severe or very severe COPD, was limited. However it is noteworthy that Siglec-1 was decreased even in the early stages of COPD patients who had never experienced acute exacerbation. Second, we did not investigate a prospective cohort to determine whether patients with COPD who have lower expression of Siglec-1 on their alveolar macrophages exhibited higher bacterial colonisation or experienced more acute exacerbations. Further functional studies with prospective larger cohorts are needed to precisely define the roles of Siglec-1 on alveolar macrophages in innate host defence under pathologic conditions. Third, as we performed the in vitro phagocytosis assay with female control never smokers, we cannot exclude a possibility that gender and smoking status might affect this result. Fourth, because all patients in this study had primary lung cancers, the presence of tumour cells might suppress the expressions of phagocytic receptors and the ability of bacterial phagocytosis. Although we used peripheral lung tissues distant from tumor lesions, recent reports have indicated that cancer cells might have a crosstalk with distant cells via extracellular vesicles to inhibit anti-tumour immunity [41]. Fifth, small numbers of dendritic cells and monocytes might be included in the phagocytosis assay, since we did not exclude those cells using specific cell surface markers in flow cytometry. Finally, although we observed decreases in expression of Fc $\gamma$ RI and MSR-1 in COPD macrophages compared to CNS, we did not determine whether Siglec-1 ${ }^{\text {dim-neg }}$ macrophages also lost expression of Fc $\gamma$ RI and MSR-1 or if decreased receptor expression in COPD alveolar macrophages had less specificity. It would be notable for a future study to evaluate multiple receptor expression of alveolar macrophages in diseased lungs.

\section{Conclusions}

We demonstrated that the extracellular expression of Siglec-1 was decreased on alveolar macrophages of COPD especially with pulmonary emphysema. Considering the involvement of Siglec-1 in NTHi phagocytosis by human alveolar macrophages, our data shed light on the importance of phagocytic receptors in alveolar macrophages in the pathogenesis of COPD. 


\section{Supplementary information}

Supplementary information accompanies this paper at https://doi.org/10. 1186/s12931-020-1297-2

\section{Additional file 1: Figure S1. CD206 is a marker for alveolar} macrophages in human lungs. A representative image of sorted FSChi, $\mathrm{SSC}^{\text {hi }} / \mathrm{CD} 45^{+} / \mathrm{CD} 26^{+}$cells by Diff-Quick stain. An error bar, $50 \mu \mathrm{m}$. Representative immunostaining for CD206 in lung tissues of control neversmokers (CNS, upper images), control ex-smokers (CES, middle images) and COPD ex-smokers (COPD, lower images). Right images are highly magnified images from each rectangle in left, corresponding images. Scale bars indicate $50 \mu \mathrm{m}$ (left images), $25 \mu \mathrm{m}$ (right images). Nuclei were counterstained with haematoxylin (blue). Histograms of CD14 expression on $\mathrm{FSC}^{\mathrm{hi}} / \mathrm{SSC}^{\mathrm{hi}} / \mathrm{CD} 45^{+} / \mathrm{CD} 206^{+}$cells and on $\mathrm{FSC}^{\mathrm{lo}} / \mathrm{SSC}^{\mathrm{lo}} / \mathrm{CD} 45^{+} / \mathrm{CD}_{20} 6^{-}$ cells from human lung tissue lavages. FSC hi $/ \mathrm{SSC}^{\text {hi }} / \mathrm{CD} 45^{+} / \mathrm{CD} 206^{+}$cells showed little expression of $\mathrm{CD} 14 . \mathrm{FSC}^{\mathrm{lo}} / \mathrm{SSC} \mathrm{lo}^{\mathrm{l}} / \mathrm{CD} 45^{+} / \mathrm{CD} 206^{-}$cells contained CD14-highly expressing cells corresponding monocytes (an arrow) $[1,2]$. Specific staining, red; isotype control, gray. Fig. S2. Siglec-1 expression in human alveolar macrophages. Representative immunostaining for Siglec-1 in lung tissues of five control never-smokers (a left image) and a negative control staining with only secondary antibody (a right image). Scale bars indicate $50 \mu \mathrm{m}$. Table S1. Antibodies for flow cytometry. Table S2. Isotype-matched control antibodies

\section{Abbreviations}

BSA: Bovine serum albumin; CES: Control ex-smokers; CNS: Control neversmokers; COPD: Chronic obstructive pulmonary disease; CT: Computed tomography; eQTLs: Genome-wide expression quantitative trait loci; FCYRI: Fcy receptor I; FEV1\%pred: \% predicted values of forced expiratory volume in 1 second; FMO: Fluorochrome minus one; GOLD: Global Initiative for Chronic Obstructive Lung Disease; IFN: Interferon; Ig: Immunoglobulin; ITAM: Immunoreceptor tyrosine-based activating motif;

ITIM: Immunoreceptor tyrosine-based inhibitory motif; MARCO: Macrophage receptor with collagenous structure; MSR-1: macrophage scavenger receptor1; NTHi: Non-typeable Haemophilus influenzae; PBS: Phosphate-buffered saline; ROS: Reactive oxygen species; SH2: Src homology 2; SNP: Single nucleotide polymorphism; Syk: Spleen tyrosine kinase

\section{Acknowledgments}

We thank the Biomedical Research Core of Tohoku University Graduate School of Medicine and the Biomedical Research Unit of Tohoku University Hospital for technical support. We very much appreciate Mr. Brent K Bell for reading of the manuscript and Ms. Takahashi for her excellent technical support.

\section{Authors' contributions}

NF had full access to all of the data in this study and take responsibility for the integrity of the data and the accuracy of the data analysis. NF, MY, HisatohiS and $\mathrm{MI}$ conceived this project, designed strategy, interpreted data and wrote the manuscript. AT, NF, TH, RT and HirohitoS performed and analyzed experiments. SS and YO contributed to lung sample collection. All authors read and approved the final manuscript.

\section{Funding}

This work was supported by the Japan Society for the Promotion of Science (JSPS) KAKENHI Grant Numbers JP17H04180, JP16K15453, JP26293195, JP16H05307.

\section{Availability of data and materials}

All of the datasets in the current study are available from the corresponding author on reasonable request.

\section{Ethics approval and consent to participate}

This study was approved by the ethics committees at Tohoku University School Graduate of Medicine (registration number: 2017-1-352) and Japanese Red Cross Ishinomaki Hospital (registration number: 13-12). Written informed consent was obtained from all subjects who participated in this study.

\section{Consent for publication}

Not applicable.

\section{Competing interests}

The authors declare that they have no competing interests.

\section{Author details}

${ }^{1}$ Department of Respiratory Medicine, Tohoku University Graduate School of Medicine, 1-1 Seiryocho, Aobaku, Sendai 980 8574, Japan. ${ }^{2}$ Department of Thoracic Surgery, Japanese Red Cross Ishinomaki Hospital, Ishinomaki 986 8522, Japan. ${ }^{3}$ Department of Thoracic Surgery, Institute of Development, Aging and Cancer, Tohoku University, Sendai 980 8575, Japan.

Received: 17 October 2019 Accepted: 16 January 2020

Published online: 28 January 2020

\section{References}

1. Hussell T, Bell TJ. Alveolar macrophages: plasticity in a tissue-specific context. Nat Rev Immunol. 2014;14:81-93.

2. Yu Y-RA, Hotten DF, Malakhau Y, Volker E, Ghio AJ, Noble PW, et al. Flow Cytometric analysis of myeloid cells in human blood, Bronchoalveolar lavage, and lung tissues. Am J Respir Cell Mol Biol. 2016;54:13-24.

3. Bharat A, Bhorade SM, Morales-Nebreda L, McQuattie-Pimentel AC, Soberanes S, Ridge K, et al. Flow Cytometry reveals similarities between lung macrophages in humans and mice. Am J Respir Cell Mol Biol. 2016;54:147-9.

4. Berenson CS, Garlipp MA, Grove L, Maloney J, Sethi S. Impaired phagocytosis of nontypeable Haemophilus influenzae by human alveolar macrophages in chronic obstructive pulmonary disease. J Infect Dis. 2006;194:1375-84

5. Taylor AE, Finney-Hayward TK, Quint JK, Thomas CMR, Tudhope SJ, Wedzicha JA, et al. Defective macrophage phagocytosis of bacteria in COPD. Eur Respir J. 2010;35:1039-47.

6. Berenson CS, Kruzel RL, Eberhardt E, Sethi S. Phagocytic dysfunction of human alveolar macrophages and severity of chronic obstructive pulmonary disease. J Infect Dis. 2013;208:2036-45.

7. Donnelly LE, Barnes PJ. Defective phagocytosis in airways disease. Chest 2012:141:1055-62.

8. Gordon S. Phagocytosis: An Immunobiologic Process. Immunity. 2016:44:463-75.

9. Flannagan RS, Jaumouillé V, Grinstein S. The cell biology of phagocytosis. Annu Rev Pathol. 2012;7:61-98.

10. Canton J, Neculai D, Grinstein S. Scavenger receptors in homeostasis and immunity. Nat Rev Immunol. 2013;13:621-34.

11. Brown GD, Crocker PR. Lectin receptors expressed on myeloid cells. Microbiol Spectr. 2016;4. https://doi.org/10.1128/microbiolspec.MCHD0036-2016.

12. Hodge S, Hodge G, Ahern J, Jersmann H, Holmes M, Reynolds PN. Smoking alters alveolar macrophage recognition and phagocytic ability: implications in chronic obstructive pulmonary disease. Am J Respir Cell Mol Biol. 2007;37:748-55

13. Löfdahl JM, Wahlström J, Sköld CM. Different inflammatory cell pattern and macrophage phenotype in chronic obstructive pulmonary disease patients, smokers and non-smokers. Clin Exp Immunol. 2006;145:428-37.

14. Vogelmeier CF, Criner GJ, Martinez FJ, Anzueto A, Barnes PJ, Bourbeau J, et al. Global strategy for the diagnosis, management, and prevention of chronic obstructive lung disease 2017 report. GOLD executive summary. Am J Respir Crit Care Med. 2017;195:557-82.

15. Goddard PR, Nicholson EM, Laszlo G, Watt I. Computed tomography in pulmonary emphysema. Clin Radiol. 1982;33:379-87.

16. Puryear WB, Akiyama H, Geer SD, Ramirez NP, Yu X, Reinhard BM, et al. Interferon-inducible mechanism of dendritic cell-mediated HIV-1 dissemination is dependent on Siglec-1/CD169. PLoS Pathog. 2013;9: e1003291.

17. Macauley MS, Crocker PR, Paulson JC. Siglec-mediated regulation of immune cell function in disease. Nat Rev Immunol. 2014:14:653-66.

18. Crocker PR, Paulson JC, Varki A. Siglecs and their roles in the immune system. Nat Rev Immunol. 2007:7:255-66.

19. Ravetch JV, Lanier LL. Immune inhibitory receptors. Science. 2000;290:84-9. 
20. Angata T, Ishii T, Motegi T, Oka R, Taylor RE, Soto PC, et al. Loss of Siglec-14 reduces the risk of chronic obstructive pulmonary disease exacerbation. Cell Mol Life Sci. 2013;70:3199-210.

21. Lamontagne M, Timens W, Hao K, Bossé Y, Laviolette M, Steiling K, et al. Genetic regulation of gene expression in the lung identifies CST3 and CD22 as potential causal genes for airflow obstruction. Thorax. 2014;69:997-1004

22. Crocker PR, Kelm S, Dubois C, Martin B, McWilliam AS, Shotton DM, et al. Purification and properties of sialoadhesin, a sialic acid-binding receptor of murine tissue macrophages. EMBO J. 1991;10:1661-9.

23. Kelm S, Brossmer R, Isecke R, Gross HJ, Strenge K, Schauer R. Functional groups of sialic acids involved in binding to siglecs (sialoadhesins) deduced from interactions with synthetic analogues. Eur J Biochem. 1998;255:663-72.

24. Crocker PR, Mucklow S, Bouckson V, McWilliam A, Willis AC, Gordon S, et al. Sialoadhesin, a macrophage sialic acid binding receptor for haemopoietic cells with 17 immunoglobulin-like domains. EMBO J. 1994;13:4490-503.

25. Klaas M, Crocker PR. Sialoadhesin in recognition of self and non-self. Semin Immunopathol. 2012;34:353-64.

26. Jones C, Virji M, Crocker PR. Recognition of sialylated meningococcal lipopolysaccharide by siglecs expressed on myeloid cells leads to enhanced bacterial uptake. Mol Microbiol. 2003;49:1213-25.

27. Heikema AP, Bergman MP, Richards H, Crocker PR, Gilbert M, Samsom JN, et al. Characterization of the specific interaction between sialoadhesin and sialylated campylobacter jejuni lipooligosaccharides. Infect Immun. 2010;78:3237-46.

28. Chang Y-C, Olson J, Louie A, Crocker PR, Varki A, Nizet V. Role of macrophage sialoadhesin in host defense against the sialylated pathogen group B Streptococcus. J Mol Med. 2014;92:951-9.

29. Klaas M, Oetke C, Lewis LE, Erwig LP, Heikema AP, Easton A, et al. Sialoadhesin promotes rapid proinflammatory and type I IFN responses to a sialylated pathogen, Campylobacter jejuni. J Immunol. 2012;189:2414-22.

30. Vimr ER, Kalivoda KA, Deszo EL, Steenbergen SM. Diversity of microbial sialic acid metabolism. Microbiol Mol Biol Rev. 2004;68:132-53.

31. Apicella MA. Nontypeable Haemophilus influenzae: the role of N-acetyl-5neuraminic acid in biology. Front Cell Infect Microbiol. 2012;2:19.

32. Sapey E, Stockley RA. COPD exacerbations . 2: aetiology. Thorax. 2006;61:250-8.

33. Moghaddam SJ, Ochoa CE, Sethi S, Dickey BF. Nontypeable Haemophilus influenzae in chronic obstructive pulmonary disease and lung cancer. Int J Chron Obstruct Pulmon Dis. 2011;6:113-23.

34. Bewley MA, Preston JA, Mohasin M, Marriott HM, Budd RC, Swales J, et al. Impaired mitochondrial Microbicidal responses in chronic obstructive pulmonary disease macrophages. Am J Respir Crit Care Med. 2017:196:845-55.

35. Harvey CJ, Thimmulappa RK, Sethi S, Kong X, Yarmus L, Brown RH, et al. Targeting Nrf2 signaling improves bacterial clearance by alveolar macrophages in patients with COPD and in a mouse model. Sci Transl Med. 2011;3:78ra32.

36. York MR, Nagai T, Mangini AJ, Lemaire R, van Seventer JM, Lafyatis R. A macrophage marker, Siglec-1, is increased on circulating monocytes in patients with systemic sclerosis and induced by type I interferons and tolllike receptor agonists. Arthritis Rheum. 2007;56:1010-20.

37. Rempel H, Calosing C, Sun B, Pulliam L. Sialoadhesin expressed on IFNinduced monocytes binds HIV-1 and enhances infectivity. PLoS One. 2008:3:e1967.

38. Fraschilla I, Pillai S. Viewing Siglecs through the lens of tumor immunology. Immunol Rev. 2017;276:178-91

39. Asano K, Nabeyama A, Miyake Y, Qiu C-H, Kurita A, Tomura M, et al. CD169positive macrophages dominate antitumor immunity by crosspresenting dead cell-associated antigens. Immunity. 2011:34:85-95.

40. Asano K, Takahashi N, Ushiki M, Monya M, Aihara F, Kuboki E, et al. Intestinal CD169(+) macrophages initiate mucosal inflammation by secreting CCL8 that recruits inflammatory monocytes. Nat Commun. 2015;6:7802.

41. Kogure A, Kosaka N, Ochiya T. Cross-talk between cancer cells and their neighbors via miRNA in extracellular vesicles: an emerging player in cancer metastasis. J Biomed Sci. 2019;26:7-8.

\section{Publisher's Note}

Springer Nature remains neutral with regard to jurisdictional claims in published maps and institutional affiliations.

\section{Ready to submit your research? Choose BMC and benefit from:}

- fast, convenient online submission

- thorough peer review by experienced researchers in your field

- rapid publication on acceptance

- support for research data, including large and complex data types

- gold Open Access which fosters wider collaboration and increased citations

- maximum visibility for your research: over $100 \mathrm{M}$ website views per year

At BMC, research is always in progress.

Learn more biomedcentral.com/submissions 